\title{
Review
}

Neuro[mmunoModulation

\section{A Review on the Role of Inflammation in Attention-Deficit/Hyperactivity Disorder}

\author{
Douglas Teixeira Leffa ${ }^{a, b}$ Iraci L.S. Torres ${ }^{a, b}$ Luis Augusto Rohde ${ }^{c, d}$ \\ a Post-Graduate Program in Medicine, Medical Sciences, School of Medicine, Universidade Federal do Rio Grande

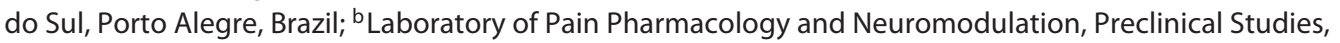 \\ Pharmacology Department, Instituto de Ciências Básicas da Saúde, Universidade Federal do Rio Grande do Sul, \\ Porto Alegre, Brazil; 'ADHD Outpatient Program, Hospital de Clínicas de Porto Alegre, Universidade Federal do Rio \\ Grande do Sul, Porto Alegre, Brazil; ${ }^{d}$ National Institute of Developmental Psychiatry for Children and Adolescents, \\ São Paulo, Brazil
}

\section{Keywords}

Attention-deficit/hyperactivity disorder · Inflammation ·

Neuronal damage $\cdot$ Biomarkers

\begin{abstract}
Attention-deficit/hyperactivity disorder (ADHD) is a prevalent neurodevelopmental condition that impairs quality of life in social, academic, and occupational contexts for both children and adults. Although a strong neurobiological basis has been demonstrated, the pathophysiology of ADHD is still poorly understood. Among the proposed mechanisms are glial activation, neuronal damage and degeneration, increased oxidative stress, reduced neurotrophic support, altered neurotransmitter metabolism, and blood-brain barrier disruption. In this way, a potential role of inflammation has been increasingly researched. However, evidence for the involvement of inflammation in ADHD is still scarce and comes mainly from (1) observational studies showing a strong comorbidity of ADHD with inflammatory and autoimmune disorders; (2) studies evaluating serum inflammatory markers; and (3) genetic studies. A co-occurrence of ADHD with inflammatory disorders has been demonstrated in a large number of subjects, suggesting a range of underlying mech-
\end{abstract}

anisms such as an altered immune response, common genetics, and environmental links. The evaluation of serum inflammatory markers has provided mixed results, likely due to the small sample sizes and high heterogeneity between biomarkers. However, there is evidence that increased inflammation during the early development may be a risk factor for ADHD symptoms. Although genetic studies have demonstrated a potential role for inflammation in this disorder, there is no clear evidence. To sum up, inflammation may be an important mechanism in ADHD pathophysiology, but more studies are still needed for a more precise conclusion.

(c) 2018 S. Karger AG, Basel

\section{Epidemiology}

Attention-deficit/hyperactivity disorder (ADHD) is a neurodevelopmental disorder characterized by the impairing symptoms of inattention and/or hyperactivity/ impulsivity [1]. A meta-analysis published in 2007 has demonstrated that the worldwide prevalence of ADHD in

Iraci L.S. Torres and Luis Augusto Rohde contributed equally to this work.

\section{KARGER}

(c) 2018 S. Karger AG, Basel 
children and adolescents is of 5.3\% (95\% CI: 5.01-5.56) [2]. In adults, ADHD has been shown to persist in about $50-70 \%$ of the cases depending on methodological issues in the studies [3, 4]. Accordingly, a meta-analysis has shown a prevalence of about $2.4 \%$ in adulthood [5]. Concurring with those results, a recent study conducted by the World Health Organization has found a prevalence of $2.5 \%$ in adults in the 30 countries evaluated [6].

\section{Impairments}

ADHD impairs the quality of life and well-being in social, academic, and occupational contexts for both children and adults. The pathology is correlated with a wide range of psychiatry comorbidities, psychological dysfunctions, and risk behaviors. Observational studies conducted in large populations have demonstrated that an ADHD diagnosis is a risk factor for substance use disorder [7], criminal behavior [8], and greater susceptibility to physical injuries [9]. A cohort study that followed 1,92 million people, 32,061 of whom having a diagnosis of ADHD, found a significant increase in the mortality rate in this population. The increase in mortality was mainly related to death caused by unnatural factors, especially accidents [10]. Adverse outcomes in adolescence and adulthood include academic and vocational underachievement [11], obesity [12], unemployment, low economic status [13], emotional dysregulation [14], and pregnancy in adolescence [15]. It has been shown that ADHD patients present higher suicide attempts when compared to the general population [16]. To sum up, ADHD has an important impact in the quality of life of those affected, comparable to other severe psychiatry disorders [17].

\section{Diagnosis}

The diagnosis of ADHD is based on the assessment of inattentive or hyperactive-impulsive symptoms. According to the Diagnostic and Statistical Manual of Mental Disorders, ed 5 (DSM-5), the diagnosis of ADHD should be performed when there is clear evidence that the symptoms impair the quality of life in social, academic, or occupational performance [1]. In addition, the symptoms should be present in 2 or more different settings, and their onset should be prior to 12 years of age. The diagnosis relies on the reporting of patients or other informants (relatives, teachers), and the clinical interview remains the gold standard.

A Review on the Role of Inflammation in Attention-Deficit/Hyperactivity Disorder
The diagnosis of ADHD involves cultural and ethnical differences related to the attitudes towards the disease. For instance, it has been reported that African American youths have more ADHD symptoms when compared to Caucasians, but the latter were diagnosed two-thirds as often [18]. This difference could be explained by parents' beliefs about ADHD and the lack of access to treatment [18]. Currently, there is no biomarker for the diagnosis of $\mathrm{ADHD}$, and the reliance only on a clinical interview may be an important factor predisposing negative cultural views on the disorder and uncertainty concerning the validity of the diagnosis [19].

\section{Neurobiological Basis}

The neurobiological basis of $\mathrm{ADHD}$ has been confirmed through genetic and neuroimaging studies [5]. Twin studies have shown that ADHD has a strong heritability of $70-80 \%$ in both children and adults [20]. According to genome-wide association studies, approximately $40 \%$ of the heritability is associated to common genetic variants [21]. In addition, candidate gene studies have mainly shown the influence of genes involved in the monoamine neurotransmitter system [22]. A recent genome-wide association meta-analysis conducted with 20,183 ADHD patients and 35,191 controls identified 12 independent loci exceeding genome-wide significance [Demontis, pers. commun.]. The biological role of the identified loci appears to be related to neural development and plasticity, neuronal wiring, dopamine levels in the synapses, intellectual disability, and the development of speech and learning [Demontis, pers. commun.]. In relation to environmental factors, severe early maternal deprivation appears to have a causal role [22]. However, other environmental factors such as maternal smoking and alcohol use, low birth weight, premature birth, and exposure to environmental toxins have also been associated with ADHD symptoms $[23,24]$.

ADHD is characterized by structural and functional dysfunctions in a wide range of cortical and subcortical regions. A recent mega-analysis performed in a sample of 1,713 patients with ADHD and 1,529 controls has demonstrated a volume reduction in the nucleus accumbens, amygdala, caudate, hippocampus, and putamen in patients with the disorder [25]. A meta-analysis of functional magnetic studies including approximately $200 \mathrm{ADHD}$ patients and 200 controls has demonstrated a reduction in the activity of distinct cortical regions during tests requiring attention and impulsivity control [26]. Although

Neuroimmunomodulation 2018;25:328-333 
a wide literature has repeatedly shown that ADHD has a strong neurobiological basis, its pathophysiology is still poorly understood. It is believed that several factors, including genetic and environmental factors, interact during development, giving rise to ADHD symptomatology.

\section{The Role of Inflammation}

Among the neuropathological mechanisms believed to be involved in ADHD, there has been a growing interest in the immune system. Over the last years, an increasing body of evidence has supported the role of inflammation in neuropsychiatric disorders. A strong association between altered inflammatory mechanisms and neuropsychiatric disorders has been provided for depression [27], schizophrenia [28], bipolar disorder [29], and posttraumatic stress disorder [30] through systematic reviews and meta-analyses. It has been hypothesized that inflammatory mechanisms are related to the physiopathology of neuropsychiatric disorders through several mechanisms. Among them are glial activation [31], neuronal damage and degeneration [32], increased oxidative stress [33], reduced neurotrophic support [34], altered neurotransmitter metabolism [35], and blood-brain barrier disruption [36]. For ADHD, evidence supporting a role of inflammatory mechanisms comes from 3 main lines: comorbidity with inflammatory and autoimmune disorders, biochemical markers, and genetic studies. In this sense, the aim of this review is to present a summary of the existing literature regarding the role of inflammation in the pathophysiology of ADHD.

\section{Comorbidity with Inflammatory and Autoimmune Disorders}

Observational data from a large number of subjects show a strong association between ADHD and inflammatory and autoimmune disorders. Miyazaki et al. [37] performed a systematic review and meta-analysis with more than 61,000 children (about 8,000 ADHD patients) to evaluate an association between $\mathrm{ADHD}$ and allergies. They found that ADHD patients were more likely to have asthma, allergic rhinitis, atopic dermatitis, and allergic conjunctivitis in comparison to the non-ADHD subjects from the population. Similar results were found by Schans et al. [38], performing a systematic review and meta-analysis examining the co-occurrence of atopy and ADHD. They found a higher presence of asthma, eczema, and rhi- nitis in ADHD patients when compared to a control population. A prospective cohort study performed with more than 23,000 patients demonstrated that a personal and a maternal history of autoimmune disease were also associated with an increased risk of ADHD [39]. Among the autoimmune diseases there were thyrotoxicosis, type 1 diabetes, autoimmune hepatitis, psoriasis, and ankylosing spondylitis. Cross-sectional studies have also identified a higher prevalence of psoriasis in ADHD patients [40].

The higher co-occurrence of ADHD with inflammatory and autoimmune disorders may suggest a range of underlying mechanisms, including an altered immune response, common genetics, and environmental links. It has also been suggested that increased cytokine release due to an inflammatory process may affect the prefrontal cortex functioning [41]. In addition, ADHD abnormalities may be the result of an exaggerated central nervous system inflammatory response in the fetus caused by maternal inflammation, such as in allergy or autoimmune diseases. The prevalent comorbidity between ADHD and inflammatory disorders may also explain the association found between ADHD diagnosis and the use of acetaminophen during pregnancy [42]. To sum up, although observational data support the co-occurrence of ADHD and inflammatory and autoimmune disorders, the studies conducted so far have not identified which factors may have a causal role in this comorbidity. For that, well-designed prospective studies are still needed.

\section{Biochemical Markers}

Studies searching for inflammatory biomarkers in ADHD patients have not provided conclusive findings, likely due to small sample sizes and a high heterogeneity among biomarkers. Inflammatory markers tested in this population include specific antibodies, cytokines, and neurotrophic factors. Passarelli et al. [43] evaluated the role of antibodies against Purkinje cells as a possible marker of an immune response in ADHD patients. These specific antibodies were chosen since a role of the cerebellum in the pathophysiology of ADHD has been previously proposed [5]. The authors found a significantly higher immunoreactivity against anti-Purkinje cell antibodies in ADHD patients when compared to controls, suggesting an involvement of the autoimmune system in the disorder. These results have been replicated in a following study [44], and the authors also demonstrated that ADHD patients had increased serum levels of interleukin 
6 (IL-6) and IL-10. Increased levels of antibasal ganglia antibodies [45] and antibodies against the dopamine transporter [46] have also been detected in ADHD, supporting the role of the immune system in the disorder.

Several cytokines have been researched as possible neurochemical markers of ADHD. As previously mentioned, there is a high heterogeneity among the biomarkers tested, which makes the interpretations of the findings more challenging. Evaluation of pro- and anti-inflammatory cytokines and the cytokine-related neurotrophin S100B have been performed by Oades et al. [47] in the serum of ADHD patients. There was no major imbalance in the levels of inflammatory markers between $\mathrm{ADHD}$ patients and controls, and there were no differences in the levels of S100B. In a second study, however, the total serum S100B levels were positively associated with reduced symptoms in patients [48]. Serum IL-6 and tumor necrosis factor alpha were evaluated in a different study, and no significant results were found [49].

Although the previously mentioned studies did not show significant main findings related to inflammatory markers, a cohort study performed with more than 1,500 premature and low-birth-weight newborns measured 25 inflammation-related proteins in the serum and found that children who had elevated concentrations of inflammation-related proteins during the first 2 postnatal weeks were more likely to have attention problems at 24 months [50]. In addition, neonatal infections, which are associated with inflammatory responses, and systemic inflammation during the first postnatal month increased the risk of ADHD [51, 52]. To sum up, the evaluation of serum levels of inflammatory markers in ADHD patients has provided mixed results, likely due to small sample sizes and a high heterogeneity between biomarkers. However, there is evidence that increased inflammation during the early development may be a risk factor for ADHD symptoms.

\section{Genetic Studies}

There has been evidence from genetic studies that polymorphisms in genes related to inflammatory pathways play a role in ADHD. Smith et al. [53] performed a study evaluating a set of 164 single-nucleotide polymorphisms (SNPs) from 31 candidate genes in a total of 398 subjects. They found that 2 SNPs in a cytokine-related gene, the ciliary neurotrophic factor receptor (CNTFR), were associated with ADHD inattentive symptom severity. A population-based association study performed in $546 \mathrm{ADHD}$ patients and 546 controls has also identified

A Review on the Role of Inflammation in

Attention-Deficit/Hyperactivity Disorder an association between the CNTFR and ADHD in both adults and children. An association of ADHD with genes of the major histocompatibility complex has been reported [54], supporting the role of inflammation and autoimmunity in the disorder. However, those findings were not replicated in a recent genome-wide association metaanalysis [Demontis, pers. commun.].

A genome-wide association study performed by Zayats et al. [55] in 478 ADHD patients and 880 controls found no SNPs at the significance threshold. However, a pathway analysis found an association with SNPs involved in the regulation of gene expression, cell adhesion, and inflammation. A study performed by de Jong et al. [56] investigated a genomic overlap between ADHD and other psychiatric disorders in 318 individuals, 93 of whom with a diagnosis of ADHD. They found a similar genetic signature between $\mathrm{ADHD}$ and depression in genes related to inflammation. Segman et al. [57] investigated the role of the IL-1 receptor antagonist gene variable number tandem repeat polymorphism in the risk of ADHD. The IL-1 was chosen since it has been shown to modulate catecholaminergic transmission in mice [58]. They evaluated a sample of 86 children with ADHD and found an association between the 4-repeat allele and an increased risk for ADHD, and an association between the 2-repeat allele and a decreased risk. However, the same results were not replicated in a larger sample [59].

Although a good number of genetic studies provide evidence on the role of inflammation in ADHD, it is important to consider that there is a high variation between the methodologies performed. In addition, there is no consensus about which inflammatory-related genes predispose to ADHD. A possible explanation for that is the fact that ADHD is a disorder with a highly heterogeneous genetic makeup and clinical presentation. Therefore, studies focusing on a more homogeneous population may be more likely to be conclusive.

\section{Conclusions}

There has been a growing interest in inflammation as a predisposing mechanism in psychiatric disorders. In ADHD, evidence from comorbidity with inflammatory and autoimmune disorders, serum biomarkers, and genetic studies can be found. The high co-occurrence between ADHD and inflammatory disorders suggests a range of underlying mechanisms, including altered immune response and common genetic and environmental links. Biomarkers measured in ADHD patients have pro- 
vided unclear results. However, increased inflammation during the early development appears to be related to an ADHD phenotype. ADHD is a highly hereditary disorder; therefore, it might be expected that polymorphisms in inflammatory-related genes are present in patients. Although some studies have found this association, there is no consensus about which genes are affected. To sum up, there have been indications for a role of the immune system in the pathophysiology of ADHD. However, well-designed studies are still needed to confirm this hypothesis.

\section{Acknowledgements}

This work was supported by the following Brazilian funding agencies: Brazilian Federal Agency for Support and Evaluation of Graduate Education - CAPES/PNPD (Teixeira Leffa D., Edital
PBE-DPM 62/2014); National Council for Scientific and Technological Development - CNPq (L.S. Torres I., Rohde L.A.); and the Graduate Research Group (GPPG) of Hospital de Clínicas de Porto Alegre - HCPA (L.S. Torres I., grant number 14-0103).

\section{Disclosure Statement}

Rohde L.A. has been a member of the speakers' bureau/ advisory board and/or acted as a consultant for Eli-Lilly, JanssenCilag, Medice, Novartis, and Shire in the last 3 years. He receives authorship royalties from Oxford Press and ArtMed. He has also received travel awards from Shire for his participation in the 2015 WFADHD and 2016 AACAP meetings. The ADHD and Juvenile Bipolar Disorder Outpatient Programs chaired by him received unrestricted educational and research support from the following pharmaceutical companies in the last 3 years: Eli-Lilly, Janssen-Cilag, Novartis, and Shire.

\section{References}

1 American Psychiatric Association: Diagnostic and Statistical Manual of Mental Disorders, ed 5. 2013.

2 Polanczyk G, de Lima MS, Horta BL, Biederman J, Rohde LA: The worldwide prevalence of ADHD: a systematic review and metaregression analysis. Am J Psychiatry 2007;164: 942-948.

3 Asherson P, Buitelaar J, Faraone SV, Rohde LA: Adult attention-deficit hyperactivity disorder: key conceptual issues. Lancet Psychiatry 2016;3:568-578.

4 Caye A, Spadini AV, Karam RG, Grevet EH, Rovaris DL, Bau CH, et al: Predictors of persistence of ADHD into adulthood: a systematic review of the literature and meta-analysis. Eur Child Adolesc Psychiatry 2016;25:11511159.

5 Faraone SV, Asherson P, Banaschewski T, Biederman J, Buitelaar JK, Ramos-Quiroga JA, et al: Attention-deficit/hyperactivity disorder. Nat Rev Dis Primers 2015;1:15020.

6 Fayyad J, Sampson NA, Hwang I, Adamowski T, Aguilar-Gaxiola S, Al-Hamzawi A, et al: The descriptive epidemiology of DSM-IV adult ADHD in the World Health Organization World Mental Health Surveys. Atten Defic Hyperact Disord 2017;9:47-65.

7 Groenman AP, Oosterlaan J, Rommelse N, Franke B, Roeyers H, Oades RD, et al: Substance use disorders in adolescents with attention deficit hyperactivity disorder: a 4-year follow-up study. Addiction 2013;108:15031511.

8 Lichtenstein P, Halldner L, Zetterqvist J, Sjolander A, Serlachius E, Fazel S, et al: Medication for attention deficit-hyperactivity disorder and criminality. N Engl J Med 2012;367: 2006-2014.
9 Dalsgaard S, Leckman JF, Mortensen PB, Nielsen HS, Simonsen M: Effect of drugs on the risk of injuries in children with attention deficit hyperactivity disorder: a prospective cohort study. Lancet Psychiatry 2015;2:702709.

10 Dalsgaard S, Ostergaard SD, Leckman JF, Mortensen PB, Pedersen MG: Mortality in children, adolescents, and adults with attention deficit hyperactivity disorder: a nationwide cohort study. Lancet 2015;385:21902196.

11 Fleming M, Fitton CA, Steiner MFC, McLay JS, Clark D, King A, et al: Educational and health outcomes of children treated for attention-deficit/hyperactivity disorder. JAMA Pediatr 2017;171:e170691.

12 Cortese S, Faraone SV, Bernardi S, Wang S, Blanco C: Adult attention-deficit hyperactivity disorder and obesity: epidemiological study. Br J Psychiatry 2013;203:24-34.

13 Biederman J, Faraone SV: The effects of attention-deficit/hyperactivity disorder on employment and household income. MedGenMed 2006;8:12.

14 Biederman J, Spencer T, Lomedico A, Day H, Petty CR, Faraone SV: Deficient emotional self-regulation and pediatric attention deficit hyperactivity disorder: a family risk analysis. Psychol Med 2012;42:639-646.

15 Ostergaard SD, Dalsgaard S, Faraone SV, Munk-Olsen T, Laursen TM: Teenage parenthood and birth rates for individuals with and without attention-deficit/hyperactivity disorder: a nationwide cohort study. J Am Acad Child Adolesc Psychiatry 2017;56:578-584. e3.
16 Man KKC, Coghill D, Chan EW, Lau WCY, Hollis C, Liddle E, et al: Association of risk of suicide attempts with methylphenidate treatment. JAMA Psychiatry 2017;74:1048-1055.

17 Danckaerts M, Sonuga-Barke EJ, Banaschewski T, Buitelaar J, Dopfner M, Hollis C, et al: The quality of life of children with attention deficit/hyperactivity disorder: a systematic review. Eur Child Adoles Psychiatry 2010; 19:83-105.

18 Miller TW, Nigg JT, Miller RL: Attention deficit hyperactivity disorder in African American children: what can be concluded from the past ten years? Clin Psychol Rev 2009;29:7786.

19 Mueller AK, Fuermaier ABM, Koerts J, Tucha L: Stigma in attention deficit hyperactivity disorder. Atten Defic Hyperact Disord 2012; 4:101-14.

20 Geschwind DH, Flint J: Genetics and genomics of psychiatric disease. Science 2015;349: 1489-1494.

21 Franke B, Faraone SV, Asherson P, Buitelaar J, Bau CH, Ramos-Quiroga JA, et al: The genetics of attention deficit/hyperactivity disorder in adults, a review. Mol Psychiatry 2012; 17:960-987.

22 Thapar A, Cooper M: Attention deficit hyperactivity disorder. Lancet 2016;387:12401250.

23 Thapar A, Cooper M, Eyre O, Langley K: What have we learnt about the causes of ADHD? J Child Psychol Psychiatry 2013;54: 3-16.

24 Franz AP, Bolat GU, Bolat H, Matijasevich A, Santos IS, Silveira RC, et al: Attention-deficit/ hyperactivity disorder and very preterm/very low birth weight: a meta-analysis. Pediatrics 2018;141:e20171645. 
25 Hoogman M, Bralten J, Hibar DP, Mennes M, Zwiers MP, Schweren LSJ, et al: Subcortical brain volume differences in participants with attention deficit hyperactivity disorder in children and adults: a cross-sectional megaanalysis. Lancet Psychiatry 2017;4:310-319.

26 Hart H, Radua J, Nakao T, Mataix-Cols D, Rubia K: Meta-analysis of functional magnetic resonance imaging studies of inhibition and attention in attention-deficit/hyperactivity disorder: exploring task-specific, stimulant medication, and age effects. JAMA Psychiatry 2013;70:185-198.

27 Howren MB, Lamkin DM, Suls J: Associations of depression with C-reactive protein, IL-1, and IL-6:a meta-analysis. Psychosom Med 2009;71:171-186.

28 Miller BJ, Buckley P, Seabolt W, Mellor A, Kirkpatrick B: Meta-analysis of cytokine alterations in schizophrenia: clinical status and antipsychotic effects. Biol Psychiatry 2011;70: 663-671.

29 Modabbernia A, Taslimi S, Brietzke E, Ashrafi M: Cytokine alterations in bipolar disorder: a meta-analysis of 30 studies. Biol Psychiatry 2013;74:15-25.

30 Passos IC, Vasconcelos-Moreno MP, Costa LG, Kunz M, Brietzke E, Quevedo J, et al: Inflammatory markers in post-traumatic stress disorder: a systematic review, meta-analysis, and meta-regression. Lancet Psychiatry 2015; 2:1002-1012.

31 Reus GZ, Fries GR, Stertz L, Badawy M, Passos IC, Barichello T, et al: The role of inflammation and microglial activation in the pathophysiology of psychiatric disorders. Neuroscience 2015;300:141-154.

32 Allan SM, Rothwell NJ: Cytokines and acute neurodegeneration. Nat Rev Neurosci 2001;2: 734-744.

33 Hassan W, Noreen H, Castro-Gomes V, Mohammadzai I, da Rocha JB, Landeira-Fernandez J: Association of oxidative stress with psychiatric disorders. Curr Pharm Des 2016;22: 2960-2974.

34 Sen S, Duman R, Sanacora G: Serum brainderived neurotrophic factor, depression, and antidepressant medications: meta-analyses and implications. Biological Psychiatry 2008; 64:527-532.

35 Kronfol Z, Remick DG: Cytokines and the brain: implications for clinical psychiatry. Am J Psychiatry 2000;157:683-694.

36 Pollak TA, Drndarski S, Stone JM, David AS, McGuire P, Abbott NJ: The blood-brain barrier in psychosis. Lancet Psychiatry 2018;5: 79-92.

37 Miyazaki C, Koyama M, Ota E, Swa T, Mlunde LB, Amiya RM, et al: Allergic diseases in children with attention deficit hyperactivity disorder: a systematic review and meta-analysis. BMC Psychiatry 2017;17:120.
38 Schans JV, Cicek R, de Vries TW, Hak E, Hoekstra PJ: Association of atopic diseases and attention-deficit/hyperactivity disorder: a systematic review and meta-analyses. Neurosci Biobehav Rev 2017;74:139-148.

39 Nielsen PR, Benros ME, Dalsgaard S: Associations between autoimmune diseases and attention-deficit/hyperactivity disorder: a nationwide study. J Am Acad Child Adolesc Psychiatry 2017;56:234-240.e1.

40 Hegvik TA, Instanes JT, Haavik J, Klungsoyr K, Engeland A: Associations between attention-deficit/hyperactivity disorder and autoimmune diseases are modified by sex: a population-based cross-sectional study. Eur Child Adolesc Psychiatry 2018;27:663-675.

41 Buske-Kirschbaum A, Schmitt J, Plessow F, Romanos M, Weidinger S, Roessner V: Psychoendocrine and psychoneuroimmunological mechanisms in the comorbidity of atopic eczema and attention deficit/hyperactivity disorder. Psychoneuroendocrinology 2013; 38:12-23.

42 Ystrom E, Gustavson K, Brandlistuen RE, Knudsen GP, Magnus P, Susser E, et al: Prenatal exposure to acetaminophen and risk of ADHD. Pediatrics 2017;140:e20163840.

43 Passarelli F, Donfrancesco R, Nativio P, Pascale E, Di Trani M, Patti AM, et al: Anti-Purkinje cell antibody as a biological marker in attention deficit/hyperactivity disorder: a pilot study. J Neuroimmunol 2013;258:67-70.

44 Donfrancesco R, Nativio P, Di Benedetto A, Villa MP, Andriola E, Melegari MG, et al: Anti-Yo antibodies in children with ADHD: first results about serum cytokines. J Atten Disord 2016.

45 Toto M, Margari F, Simone M, Craig F, Petruzzelli MG, Tafuri S, et al: Antibasal Ganglia Antibodies and antistreptolysin $\mathrm{O}$ in noncomorbid ADHD. J Atten Disord 2015;19:965970.

46 Giana G, Romano E, Porfirio MC,D’Ambrosio R, Giovinazzo S, Troianiello M, et al: Detection of auto-antibodies to DAT in the serum: interactions with DAT genotype and psychostimulant therapy for ADHD. J Neuroimmunol 2015;278:212-222.

47 Oades RD, Dauvermann MR, Schimmelmann BG, Schwarz MJ, Myint AM: Attention-deficit hyperactivity disorder (ADHD) and glial integrity: S100B, cytokines and kynurenine metabolism - effects of medication. Behav Brain Funct 2010;6:29.

48 Oades RD, Myint AM, Dauvermann MR, Schimmelmann BG, Schwarz MJ: Attentiondeficit hyperactivity disorder (ADHD) and glial integrity: an exploration of associations of cytokines and kynurenine metabolites with symptoms and attention. Behav Brain Funct 2010;6:32.
49 Corominas-Roso M, Armario A, Palomar G Corrales M, Carrasco J, Richarte V, et al: IL-6 and TNF-alpha in unmedicated adults with ADHD: relationship to cortisol awakening response. Psychoneuroendocrinology 2017;79: 67-73.

50 O'Shea TM, Joseph RM, Kuban KC, Allred EN, Ware J, Coster T, et al: Elevated blood levels of inflammation-related proteins are associated with an attention problem at age 24 mo in extremely preterm infants. Pediatric Res 2014;75:781-787.

51 Rand KM, Austin NC, Inder TE, Bora S, Woodward LJ: Neonatal infection and later neurodevelopmental risk in the very preterm infant. J Pediatr 2016;170:97-104.

52 Allred EN, Dammann O, Fichorova RN, Hooper SR, Hunter SJ, Joseph RM, et al: Systemic inflammation during the first postnatal month and the risk of attention deficit hyperactivity disorder characteristics among 10 year-old children born extremely preterm. J Neuroimmune Pharmacol 2017;12:531-543.

53 Smith TF, Anastopoulos AD, Garrett ME, Arias-Vasquez A, Franke B, Oades RD, et al: Angiogenic, neurotrophic, and inflammatory system SNPs moderate the association between birth weight and ADHD symptom severity. Am J Med Genet B Neuropsychiatr Genet 2014;165B:691-704.

54 Odell JD, Warren RP, Warren WL, Burger RA, Maciulis A: Association of genes within the major histocompatibility complex with attention deficit hyperactivity disorder. Neuropsychobiology 1997;35:181-186.

55 Zayats T, Athanasiu L, Sonderby I, Djurovic S, Westlye LT, Tamnes CK, et al: Genomewide analysis of attention deficit hyperactivity disorder in Norway. PloS One 2015; 10:e0122501.

56 de Jong S, Newhouse SJ, Patel H, Lee S, Dempster D, Curtis C, et al: Immune signatures and disorder-specific patterns in a cross-disorder gene expression analysis. Br J Psychiatry 2016; 209:202-208

57 Segman RH, Meltzer A, Gross-Tsur V, Kosov A, Frisch A, Inbar E, et al: Preferential transmission of interleukin-1 receptor antagonist alleles in attention deficit hyperactivity disorder. Mol Psychiatry 2002;7:72-74.

58 Lacosta S, Merali Z, Anisman H: Influence of interleukin-1beta on exploratory behaviors, plasma ACTH, corticosterone, and central biogenic amines in mice. Psychopharmacology 1998;137:351-361.

59 Misener VL, Schachar R, Ickowicz A, Malone M, Roberts W, Tannock R, et al: Replication test for association of the IL-1 receptor antagonist gene, IL1RN, with attention-deficit/ hyperactivity disorder. Neuropsychobiology 2004;50:231-234. 